\title{
Proactive variant effect mapping to accelerate genetic diagnosis for pediatric cardiac arrest
}

Brendan J. Floyd, M.D., Ph.D. ${ }^{* 1}$, Jochen Weile, Ph.D. ${ }^{* 2,3,4}$, Prince J. Kannankeril, M.D. ${ }^{5}$, Andrew M. Glazer, Ph.D. ${ }^{6}$, Chloe M. Reuter, M.S., L.C.G.C. ${ }^{7}$, Calum A. MacRae, M.D., Ph.D. ${ }^{8}$, Euan A. Ashley, FRCP, D.Phil. ${ }^{* 7,9}$, Dan M. Roden, M.D. ${ }^{* 6,10,11,12}$, Frederick P. Roth, Ph.D. ${ }^{* 2,3,4}$, and Victoria N. Parikh, M.D. ${ }^{* 7}$, for the CardioVar Consortium

1. Division of Pediatric Cardiology, Stanford University, Stanford, CA, USA.

2. Lunenfeld-Tanenbaum Research Institute, Sinai Health, Toronto, Ontario, Canada.

3. The Donnelly Centre, University of Toronto, Toronto, Ontario, Canada.

4. Departments of Molecular Genetics and Computer Science, University of Toronto, Toronto, Ontario, Canada.

5. Center for Pediatric Precision Medicine, Department of Pediatrics, Vanderbilt University Medical Center, Nashville, TN, USA.

6. Division of Clinical Pharmacology, Department of Medicine, Vanderbilt University Medical Center, Nashville, TN, USA.

7. Center for Inherited Cardiovascular Disease, Division of Cardiovascular Medicine, Stanford University, Stanford, CA, USA.

8. Department of Medicine, Brigham and Women's Hospital, Harvard Medical School, Boston, MA, USA.

9. Department of Genetics, Stanford University, Stanford, CA, USA.

10. Division of Cardiovascular Medicine, Department of Medicine, Vanderbilt University Medical Center, Nashville, TN, USA.

11. Department of Pharmacology, Vanderbilt University Medical Center, Nashville, TN, USA.

12. Department of Biomedical Informatics, Vanderbilt University Medical Center, Nashville, TN, USA.

* These authors contributed equally.

\section{Disclosures}

VNP is an SAB member and receives research support from BioMarin, Inc.

FPR is an SAB member and shareholder of SeqWell, Inc, an SAB member of BioSymetrics, Inc. and

Constantiam Inc., and a shareholder of Ranomics, Inc.

CMR is a consultant for My Gene Counsel.

EAA is a founder of Personalis, Inc and member of the AstraZeneca board of directors

\section{Correspondence to}

Victoria N. Parikh, MD

vparikh@stanford.edu

\section{Abstract}

While genetic testing is becoming standard of care for patients with potentially inherited cardiovascular disease, the prevalence of uncertain results severely limits its utility. One promising approach is to generate variant effect maps that report the function of all possible variants in a gene prospectively. The proactive clinical application of these maps is nascent, and requires careful integration with current American College of Medical Genetics guidelines for variant interpretation. Here, we describe three pediatric cases of cardiac arrest or sudden cardiac death with variants of uncertain significance in calmodulin genes. We demonstrate the prospective clinical utility of a calmodulin variant effect map to inform variant interpretation, and therefore diagnosis and family care, in each case. This study was approved by the Stanford University and Vanderbilt University Medical Center IRBs. Consent was waived based on low risk of de-identified retrospective data collection per the IRB.

\section{Keywords}

cardiovascular genetics; variant effect map; cardiac arrest 


\section{Introduction}

While genetic testing is becoming mainstream for the management of cardiovascular disease, as many as $80 \%$ variants discovered on panel testing are adjudicated as variants of uncertain significance (VUS), which cannot be used with high confidence in clinical diagnosis or family screening. ${ }^{1}$ Current variant interpretation guidelines from the American College of Medical Genetics and Genomics (ACMG) rely on high confidence evidence from kindred, population, and functional studies. ${ }^{2}$ Conventionally, investigation of variant function is performed reactively in response to identification of a novel suspicious variant in a patient. However, given recent appreciation that virtually all possible missense variants compatible with life already exist in the human population, ${ }^{3}$ this reactive approach represents a significant barrier to the flow of information in clinical genetics and its application in clinical care. ${ }^{4}$ Here, we describe the implementation of a proactive strategy to functional variant evaluation: "variant effect maps" can establish the functions of large numbers of variants prospectively, even before they are observed clinically. ${ }^{5,6}$ We present the first proactive clinical use of this functional variant effect mapping strategy, in particular for cardiovascular medicine, illustrating its promise in overcoming the VUS barrier toward widespread implementation of genomic medicine.

\section{Results}

Case 1 was an otherwise healthy pre-teen boy who presented with ventricular fibrillation (VF) arrest preceded by anxiety. After defibrillation he had polymorphic ventricular tachycardia (VT) followed by return of spontaneous circulation with sinus rhythm. EKG two days after arrest showed a prolonged QTc interval (QTcB $525 \mathrm{~ms}$ ) and he had ventricular ectopy in bigeminy on exercise testing (Figure $1 \mathrm{A \& B}$ ). He was treated with nadolol and underwent defibrillator implantation. Clinical genetic testing identified an allele with two in cis VUS in CALM2 (c.239C>G (p.Thr80Arg) and c.248A $>C$ (p.Glu83Ala)), neither of which were present in population databases. Parental genotype was unknown. Additional VUSs were identified in ANK2 and TECRL, but these variants were considered less likely to be the cause based on in silico analysis, inheritance patterns, and ClinGen curation. ${ }^{7}$

To resolve clinical equipoise surrounding the pathogenicity of the suspicious CALM2 variants, we examined a missense variant effect map of the CALM1, CALM2, and CALM3 genes, all of which encode the same calmodulin protein ${ }^{8,9,10}$ (yeast fitness screen data available on MaveDB ${ }^{5}$

https://mavedb.org/scoreset/urn:mavedb:00000001-c-2/). By comparing each variant's map score with those of known pathogenic and benign variants, we derived a log-likelihood-ratio (LLR) score representing the strength of evidence the map provides for or against pathogenicity (Figure 1C, see supplement for detailed methods). Both CALM2 variants had high LLRs, indicating a high likelihood of pathogenicity, although these alleles have not been studied in cis in this variant effect map (Figure 1C, inset). Based on the map and the overlap in patient phenotype with known calmodulin-related disease, this allele was deemed contributory to the patient's phenotype.

Case 2 was a female child with a VF arrest after a fall. Post-arrest electrocardiogram showed a QTc of $547 \mathrm{~ms}$. Clinical genetic testing identified a heterozygous VUS in CALM3 (c.281A>C; p.Asp94Ala) that had not been observed in population databases. The variant was upgraded to pathogenic only after parental testing confirmed its de novo status. We applied the CALM variant effect map to determine if it could have facilitated variant interpretation in real time, even before parental testing was complete. Indeed, the map shows a score in the range of other pathogenic variants (Figure 1C, inset).

Case 3 was a teenaged male with sudden death during sleep. His autopsy identified dilated cardiomyopathy based on chamber size and histopathologic myocardial fibrosis. Molecular autopsy revealed a VUS in CALM1, c.394G >A (p.Asp132Asn) which was not observed in ClinVar or population databases. No other candidate variants were identified. Family history was significant for diagnoses of hypertrophic cardiomyopathy in a paternal second degree relative and sudden death in a paternal third degree relative (Figure 1D). Parental testing demonstrated that the CALM1 variant was de novo, at which point the variant was upgraded to pathogenic by the lab (Figure 1D). However, the variant effect map shows a log likelihood ratio near zero, a score in the uncertain range where there is an equal prevalence of pathogenic and benign variants (Figure 1C, inset). In the setting of the patient's cardiomyopathy, which is not a recognized feature of CALM-related disease, and the family history of cardiomyopathy and sudden death, these results serve to underscore the possibility that this de novo variant is not solely responsible for this patient's sudden cardiac death. Unlike the binary assessment offered by extant ACMG criteria alone, the quantitative scores produced by the map may 
more accurately represent the risk associated with this variant, especially considering the possibility of other modifying factors or variants. Together, these data suggest that another cause of cardiomyopathy may coexist in family members, who should be clinically screened accordingly.

\section{Discussion}

These cases reveal the potential of variant effect maps to refine the use of cardiovascular genetics to facilitate patient diagnoses. The CALM map provided evidence toward a reclassification of VUSs for two of three patients. In the third case, it reinforced the lack of clinical evidence for a CALM3 variant as the sole cause of sudden cardiac arrest in the setting of cardiomyopathy. The impact of refined variant interpretation incorporating variant effect map data in the cases presented here is significant. As genetic testing is increasingly incorporated clinically in cases such as this, and indeed beyond the clinic to other sectors of society, improvements in this process will remain critical. A recent striking impact of genetic diagnosis in casting doubt on an infanticide conviction is just one example of this, where the map we use here predicts a high likilhood of pathogenicity for a CALM2 VUS found in the associated cardiac arrests (c.340G>A, p.Gly114Arg). ${ }^{11,12}$

As each of the variants discussed here was newly described, there were no functional or population data available except for the variant effect map. Without the map, the laboratory reclassification of the variant from Case 2 was fully dependent on parental sequencing: these data are costly, time intensive, and not always available clinically. Further, most variant effect maps to date are created in systems that are ancestry-agnostic (e.g. yeast or immortalized cell lines). Therefore, unlike population frequency data, variant effect map application is not dependent on patient ancestry, though the relative effect magnitude may vary across individual ancestral admixtures and across assays of variant effect.

Because these maps provide biological data at a much larger scale than previous functional studies, they represent a new class of information that will both require and allow special consideration for proper incorporation into ACMG variant interpretation guidelines. Further, as Case 3 exemplifies, while ACMG variant interpretation relies on reduction of complex data to binary risk estimates, variant effect map data are inherently quantitative and compatible with a Bayesian approach to variant classification. The continuous measurement of functional outcomes in variant effect maps may enable more precise quantification of socalled modifiers, variants with small effect sizes that increase the likelihood of disease onset, but are not sufficient to cause disease by themselves. Taken together, these reports demonstrate that prospectively generated multidimensional variant effect maps offer an opportunity to refine current approaches to variant classification, and thereby accelerate the incorporation of genetic testing in care. ${ }^{13}$ 


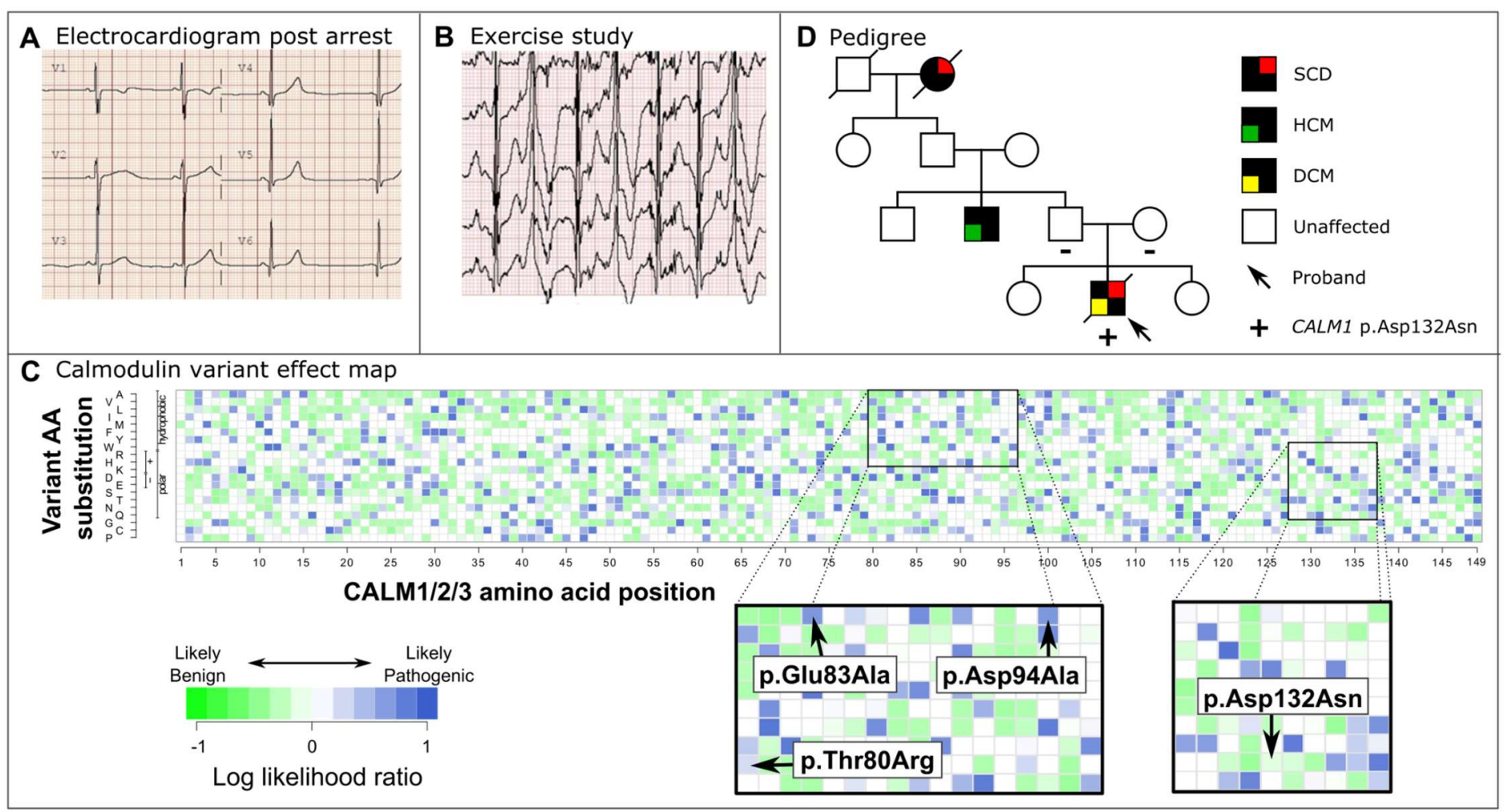

Figure 1. Proactive functional genomics for variant interpretation in CALM1/2/3. (A) EKG demonstrating long QT (525 ms) in Case 1. (B) Ventricular ectopy consistent with catecholaminergic polymorphic tachycardia at peak exercise in Case 1. (C) Variant effect map of CALM1/2/3 adapted from Weile et al. in terms of LLR, with insets indicating likelihood of pathogenicity for variants evaluated in this report. (D) Pedigree demonstrating complex family history of cardiomyopathy and sudden death despite de novo status of suspicious CALM1 VUS in Case 3. LLR, log-likelihood ratio; SCD, sudden cardiac death; HCM, hypertrophic cardiomyopathy; DCM, dilated cardiomyopathy.

\section{References}

1. van Lint FHM, Mook ORF, Alders M, Bikker H, Lekanne Dit Deprez RH, Christiaans I. Large nextgeneration sequencing gene panels in genetic heart disease: yield of pathogenic variants and variants of unknown significance. Neth Heart J. 2019;27:304-309.

2. Richards S, Aziz N, Bale S, Bick D, Das S, Gastier-Foster J, Grody WW, Hegde M, Lyon E, Spector E, Others. Standards and guidelines for the interpretation of sequence variants: a joint consensus recommendation of the American College of Medical Genetics and Genomics and the Association for Molecular Pathology. Genet Med. 2015;17:405-423.

3. Weile J, Roth FP. Multiplexed assays of variant effects contribute to a growing genotype-phenotype atlas. Hum Genet. 2018;137:665-678.

4. Green ED, Gunter C, Biesecker LG, Di Francesco V, Easter CL, Feingold EA, Felsenfeld AL, Kaufman DJ, Ostrander EA, Pavan WJ, Phillippy AM, Wise AL, Dayal JG, Kish BJ, Mandich A, Wellington CR, Wetterstrand KA, Bates SA, Leja D, Vasquez S, Gahl WA, Graham BJ, Kastner DL, Liu P, Rodriguez LL, Solomon BD, Bonham VL, Brody LC, Hutter CM, Manolio TA. Strategic vision for improving human health at The Forefront of Genomics. Nature. 2020;586:683-692.

5. Esposito D, Weile J, Shendure J, Starita LM, Papenfuss AT, Roth FP, Fowler DM, Rubin AF. MaveDB: an open-source platform to distribute and interpret data from multiplexed assays of variant effect. Genome Biol. 2019;20:223.

6. Starita LM, Ahituv N, Dunham MJ, Kitzman JO, Roth FP, Seelig G, Shendure J, Fowler DM. Variant 
Interpretation: Functional Assays to the Rescue. Am J Hum Genet. 2017;101:315-325.

7. Adler A, Novelli V, Amin AS, Abiusi E, Care M, Nannenberg EA, Feilotter H, Amenta S, Mazza D, Bikker H, Sturm AC, Garcia J, Ackerman MJ, Hershberger RE, Perez MV, Zareba W, Ware JS, Wilde AAM, Gollob MH. An International, Multicentered, Evidence-Based Reappraisal of Genes Reported to Cause Congenital Long QT Syndrome. Circulation. 2020;141:418-428.

8. Weile J, Sun S, Cote AG, Knapp J, Verby M, Mellor JC, Wu Y, Pons C, Wong C, van Lieshout N, Yang F, Tasan M, Tan G, Yang S, Fowler DM, Nussbaum R, Bloom JD, Vidal M, Hill DE, Aloy P, Roth FP. A framework for exhaustively mapping functional missense variants. Mol Syst Biol. 2017;13:957.

9. Wu Y, Weile J, Cote AG, Sun S, Knapp J, Verby M, Roth FP. A web application and service for imputing and visualizing missense variant effect maps. Bioinformatics. 2019;35:3191-3193.

10. Weile J, Kishore N, Sun S, Maaieh R, Verby M, Li R, Fotiadou I, Kitaygorodsky J, Wu Y, Holenstein A, Bürer C, Blomgren L, Yang S, Nussbaum R, Rozen R, Watkins D, Gebbia M, Kozich V, Garton M, Froese DS, Roth FP. Shifting landscapes of human MTHFR missense-variant effects. Am J Hum Genet. 2021;108:1283-1300.

11. Brohus M, Arsov T, Wallace DA, Jensen HH, Nyegaard M, Crotti L, Adamski M, Zhang Y, Field MA, Athanasopoulos V, Baró I, Ribeiro de Oliveira-Mendes BB, Redon R, Charpentier F, Raju H, DiSilvestre D, Wei J, Wang R, Rafehi H, Kaspi A, Bahlo M, Dick IE, Chen SRW, Cook MC, Vinuesa CG, Overgaard MT, Schwartz PJ. Infanticide vs. inherited cardiac arrhythmias. Europace. 2021;23:441-450.

12. The Hon Reginald Oliver Blanch AM QC. Report of the Inquiry Into the Convictions of Kathleen Megan Folbigg. Inquiry into the convictions of Kathleen Megan Folbigg; 2019.

13. Tavtigian SV, Greenblatt MS, Harrison SM, Nussbaum RL, Prabhu SA, Boucher KM, Biesecker LG, ClinGen Sequence Variant Interpretation Working Group (ClinGen SVI). Modeling the ACMG/AMP variant classification guidelines as a Bayesian classification framework. Genet Med. 2018;20:1054-1060. 